\title{
Relação entre esperança e carreira: uma revisão sistemática da literatura
}

\author{
Cássia Ferraza Alves ${ }^{l}$ \\ Clarissa Tochetto de Oliveira \\ Ana Cristina Garcia Dias \\ Marco Antônio Pereira Teixeira \\ Universidade Federal do Rio Grande do Sul, Porto Alegre-RS, Brasil
}

\section{Resumo}

Este estudo tem por objetivo caracterizar estudos empíricos que relacionaram esperança e carreira nos últimos 10 anos. Foi realizada uma revisão sistemática da literatura com as palavras-chave hope/esperança combinadas com career/carreira, work/trabalho, profession/profissão, career development/desenvolvimento de carreira, occupation/ ocupação em cinco bases de dados. Foram identificados 124 artigos, sendo 18 analisados na íntegra a partir das seguintes categorias: definição teórica de esperança e instrumentos utilizados, participantes e delineamento dos estudos, variáveis estudadas e principais resultados. A partir dos resultados, é possível identificar que esperança é uma variável importante no desenvolvimento de carreira em diferentes etapas do ciclo vital, o que indica a relevância de seu estudo e a construção de intervenções.

Palavras-chave: esperança, orientação vocacional, trabalho

\section{Abstract: Relationship between hope and career: a systematic literature review}

This study aims to characterize empirical studies that have related hope and career in the last 10 years. A systematic review of the literature was carried out with the combined keywords hope / esperança, career / carreira, work / trabalho, profession / profissão, career development / desenvolvimento de carreira, occupation / ocupação, in five databases. 124 articles were identified, 18 of which were analyzed in the following categories: theoretical definition of hope and instruments used, participants and study design, variables studied and main results. From the results, it is possible to identify that hope is an important variable in career development at different stages of the life cycle, indicating the relevance of its study and the construction of interventions.

Keywords: hope, career, work

\section{Resumen: Relación entre esperanza y carrera: una revisión sistemática de la literatura}

Este estudio tiene como objetivo caracterizar investigaciones empíricas de los últimos 10 años que han abordado la relación esperanza y carrera. Se realizó una revisión sistemática de la literatura con las palabras clave hopel esperanza combinadas con career/carrera, work/trabajo, profession/profesión, career development/desarrollo de carrera, occupation/ocupación, en cinco bases de datos. Se identificaron 124 artículos, analizándose 18 integralmente a partir de las siguientes categorías: definición teórica de esperanza e instrumentos utilizados, participantes y diseño de los estudios, variables estudiadas y principales resultados. Por medio de los resultados, es posible identificar que la variable esperanza es importante en el desarrollo de la carrera en diferentes etapas del ciclo vital, lo que evidencia la relevancia de su estudio y la propuesta de intervenciones.

Palabras clave: esperanza, carrera, trabajo

\footnotetext{
${ }^{1}$ Endereço para correspondência: Rua Ramiro Barcelos, 2600, Rio Branco, 90035-003, Porto Alegre, RS.E-mail: cassiferrazza@gmail.com
} 
O cenário atual do mercado de trabalho requer que os indivíduos estejam atentos e preparados para as constantes mudanças que podem interferir na sua carreira. Refletir sobre os planos profissionais de forma sistematizada permite traçar metas, dividir em etapas previstas e estrategicamente mon $\neg$ tadas (Lima-Dias \& Soares, 2012). No entanto, sugerir a algumas pessoas delinear planos profissionais pode ser algo angustiante, pois, diferente do século XX, as perspectivas profissionais atualmente são menos definidas e previsíveis, ou seja, apresentam transições mais frequentes. Ao mesmo tempo em que é importante delimitar metas durante o desenvolvimento de carreira, as mudanças atuais exigem dos trabalhadores o desenvolvimento de habilidades e competências para enfrentar as transições e aprimorar o gerenciamento de suas carreiras. Logo, torna-se relevante que o indivíduo antecipe mudanças, desenvolva percepção de controle e responsabilidade pelas suas escolhas de carreira (Savickas et al., 2009).

Neste sentido, o constructo esperança parece auxiliar os indivíduos na construção e no planejamento de suas carreiras (Niles, 2011). Esse tem sido estudado desde a década de 60 (Snyder et al., 1991; Snyder, 2002). Inicialmente, a esperança era compreendida como um constructo unidimensional que envolvia somente uma percepção positiva frente à possibilidade de atingir objetivos desejados. Esta expectativa influenciava nos comportamentos e na saúde do indivíduo (Snyder et al., 1991). Em 1991, Snyder et al. (1991) definiram que a esperança era composta por dois fatores inter-relacionados: agência e rotas.

Em 2002, Snyder adicionou metas ao construto esperança. Dessa forma, atualmente, a esperança é composta pelos fatores metas, rotas e agência. As metas e as rotas são os componentes cognitivos e a agência, o motivacional. As metas são os objetivos delineados pelo indivíduo. As rotas ou caminhos envolvem a percepção de sentir-se capaz de elaborar rotas/ caminhos para atingir as metas (Snyder, 2002; Snyder et al., 1991). A agência está relacionada a uma percepção de sucesso frente aos objetivos determinados (Snyder et al., 1991) e envolve a habilidade para fazer escolhas e planos de ação de forma intencional, além de motivar e regular a sua execução (Bandura, 2001).

A esperança tem sido frequentemente relacionada à identidade vocacional (Amundson, Niles, Yoon, Smith, \& Mills, 2013). Observa-se que quanto mais clara é a percepção do indivíduo sobre que profissional ele é, mais claras tendem a ser suas metas profissionais (Amundson et al., 2013). No caso de estudantes universitários, o desempenho acadêmico pode ser a rota para atingir um objetivo profissional. Estudantes com maiores níveis de esperança tendem a apresentar melhor desempenho acadêmico. Essa relação pode ser explicada pelo maior número de rotas para atingir objetivos profissionais, estratégias de estudo e de preparação para as avaliações mais adequadas, além da maior motivação para transpor eventuais obstáculos impostos pelo contexto acadêmico (Amundson et al., 2013; Onwuegbuzie \& Snyder, 2000; Seirup \& Rose, 2011; Snyder, 2002; Snyder et al., 2002).

Outra variável estudada relacionada à esperança encontrada na literatura é o comportamento exploratório (Hirschi, 2014; Hirschi et al., 2015; Sung et al., 2013). Este é definido como a busca de informações sobre si mesmo e sobre o ambiente, de forma comportamental ou reflexiva. O comportamento exploratório voltado para a carreira refere-se à exploração orientada para o trabalho como, por exemplo, a busca por um estágio extracurricular (Jordaan, 1963). Planejar o futuro, juntamente com o estabelecimento de metas, tende a estar relacionado à motivação do indivíduo para engajar-se em comportamentos exploratórios. Parece ser mais fácil para indivíduos com maiores níveis de esperança explorar várias opções relacionadas à carreira (exploração do ambiente) assim como refletir sobre seus interesses e o quanto eles estão relacionados ao trabalho (exploração de si) (Hirschi, 2014; Hirschi et al., 2015).

A esperança também tem sido relacionada com o constructo adaptabilidade de carreira, uma vez que ambos os conceitos envolvem orientação para o futuro. A adaptabilidade de carreira é entendida como a prontidão e os recursos que o indivíduo dispõe para lidar com novos desafios e transições de carreira (Savickas, 1997). Ter metas estabelecidas, apresentar rotas para atingi-las e motivação para alcançá-las pode estar relacionada à percepção de que o indivíduo é responsável por sua carreira e que apresenta a confiança de que é possível atingir as metas almejadas (Santilli et al., 2014; Wilkins et al., 2014). Esta confiança pode estar relacionada à autoeficácia, que se refere à percepção do indivíduo sobre suas próprias capacidades de empregar planos de ação e atingir os objetivos propostos (Bandura, 1977). A autoeficácia, por sua vez, também tem sido relacionada à esperança (Feldman \& Kubota, 2015; Hirschi, 2014; Hirschi et al., 2015; Sung et al., 2013) possivelmente devido ao fato de que indivíduos com mais esperança tendem a desenvolver planos e metas de carreira acreditando na sua capacidade para conduzi-los (Hirschi, 2014).

Os estudos que investigam a relação da esperança com variáveis de carreira indicam que a esperança é um constructo útil no campo do desenvolvimento e 
aconselhamento de carreira, especialmente quando se considera que, atualmente, as transições profissionais são mais frequentes. De fato, o mundo atual requer que o indivíduo se preocupe com sua carreira e com seu futuro, além de estar refletindo criticamente e se responsabilizando pelas escolhas profissionais que deve realizar ao longo da vida (Savickas et al., 2009; Savickas, 2013). Estudos demonstram que indivíduos com maiores níveis de esperança tendem a apresentar metas específicas, ter rotas plausíveis e maior confiança para empregar comportamentos a fim de atingir as metas. Eles também demonstram ser mais flexíveis frente aos desafios ao planejar novas formas de resolver problemas (Juntunen \& Wettersten, 2006; Niles, 2011; Snyder, 2002).

Diante da importância da esperança para o desenvolvimento de carreira, este estudo tem por objetivo caracterizar estudos empíricos que relacionaram esperança e carreira, a partir de uma revisão sistemática da literatura. Estudos desta natureza podem auxiliar a identificar as amostras, instrumentos, metodologias e variáveis relacionadas, a fim de avançar no desenvolvimento de estudos e intervenções que articulem teoria e prática na área de planejamento e desenvolvimento de carreira (Bernes, Bardick, \& Orr, 2007; Whiston, 2011).

\section{Método}

A revisão sistemática de literatura realizada considerou os últimos 10 anos de artigos publicados (período de 2006 e 2016), e foi realizada por duas etapas. Na primeira etapa, foram selecionadas as bases de dados investigadas: Science Direct, EBSCO, PsycInfo/Articles, Scientific Eletronic Library Online (Scielo) e Lilacs. Tais bases possibilitam localizar artigos nacionais e internacionais sobre o tema. As duas primeiras bases de dados foram escolhidas tendo em vista serem bases de dados multidisciplinares. O PsycInfo foi selecionado por ser uma base de dados da Psciologia, organizada pela American Psychological Association (APA). As bases Scielo e Lilacs foram selecionadas a fim de levantar os artigos produzidos no Brasil, América Latina e Caribe (Costa \& Zoltowski, 2014). As palavras-chave utilizadas foram "hope and career", "hope and work", "hope and profession", "hope and career development", "hope and occupation", e seus respectivos em português: "esperança e carreira", "esperança e trabalho" e "esperança e profissão", "esperança e desenvolvimento de carreira", "esperança e ocupação". As palavras-chave foram selecionadas a fim ampliar a possibilidade de selecionar publicações envolvendo esperança e carreira. Foram encontrados 124 artigos. Após a exclusão dos artigos repetidos, restaram 79 para análise do resumo. A segunda etapa consistiu na revisão dos resumos por dois juízes, pesquisadores e familiarizados com a temática analisada, com base nos seguintes critérios de inclusão: (a) estar nos idiomas: Inglês, Português ou Espanhol; (b) abordar o constructo da esperança associado à carreira, em contextos educacionais ou laborais; (c) ser um trabalho empírico. O índice de concordância entre os juízes nessa etapa foi de $94 \%$. Nesta etapa, artigos de validação, tradução e adaptação de instrumentos, assim como os de revisão teórica não foram incluídos. Optou-se por analisar somente artigos empíricos, pois esse tipo de artigo descreve claramente a metodologia do estudo e apresenta o foco nas variáveis alvo, carreira e esperança, isso difere de artigos adaptação e validação de instrumentos, que se ocupam de apresentar as propriedades psicométricas dos instrumentos. Restaram 25 artigos para análise do texto completo, após avaliação dos resumos conforme os critérios adotados. Desse modo, para análise do texto completo, foram considerados os seguintes critérios, que originaram as categorias apresentadas nos resultados e discussão: (a) buscou-se compreender a definição teórica da esperança utilizada nas pesquisas, a fim de identificar quais autores e modelos têm sido mais utilizados para subsidiar as pesquisas envolvendo a temática esperança; (b) levantar os instrumentos adotados nos estudos selecionados, considerando a existência de instrumentos com evidências de validade e a definição teórica da esperança utilizada para a construção dos mesmos; (c) identificar a etapa do desenvolvimento de carreira que os participantes dos estudos estavam vivenciando e as evidências das contribuições da esperança na relação com as variáveis estudadas nos diferentes públicos, a partir da análise dos resultados e discussão dos estudos; (d) identificar o delineamento dos estudos a fim averiguar a contribuição de estudos de outra natureza para compreensão da relação entre esperança e carreira. Somente 18 artigos possuíam texto completo disponível dos 24 artigos selecionados para a análise. A Figura 1 apresenta as etapas relatadas.

\section{Resultados e Discussão}

Os textos completos dos estudos selecionados foram analisados, sendo construídas as seguintes categorias, formuladas a priori: (a) definição conceitual de esperança e instrumentos utilizados; (b) participantes e delineamento dos estudos, (c) variáveis estudadas e principais resultados. Alguns destes resultados são apresentados de maneira sintetizada na Tabela 1. 


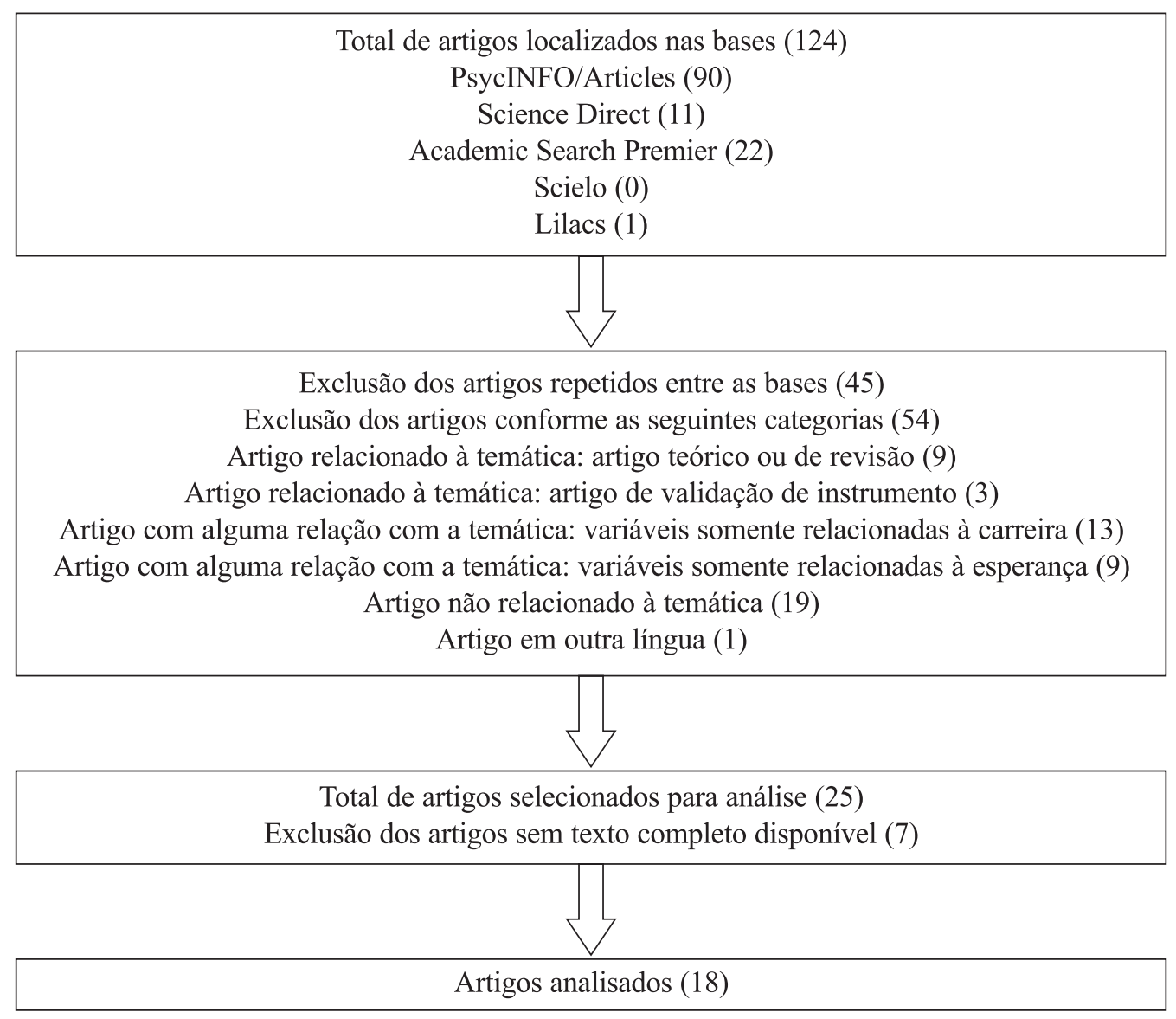

Figura 1. Etapas da revisão sistemática de literatura

Tabela 1

Objetivo, delineamento, participantes e principais resultados dos estudos da revisão sistemática

\begin{tabular}{|c|c|c|c|c|}
\hline Autores (ano) & Objetivo & Delineamento & Participantes & Principais resultados \\
\hline $\begin{array}{l}\text { Buyukgoze-Kavas } \\
\text { (2016). }\end{array}$ & $\begin{array}{l}\text { Avaliar se resiliência, } \\
\text { esperança e otimismo } \\
\text { predizem adaptabilidade } \\
\text { de carreira. }\end{array}$ & $\begin{array}{l}\text { Estudo } \\
\text { quantitativo } \\
\text { transversal. }\end{array}$ & $\begin{array}{l}415 \\
\text { universitários. }\end{array}$ & $\begin{array}{l}\text { Resiliência, esperança e otimismo } \\
\text { apresentaram correlação } \\
\text { estatisticamente significativa e } \\
\text { moderada com a adaptabilidade de } \\
\text { carreira. Isso sugere que jovens que } \\
\text { são mais resilientes, com esperança } \\
\text { e otimistas tendem a apresentar } \\
\text { mais adaptabilidade na carreira. } \\
\text { A adaptabilidade foi predita pela } \\
\text { esperança, resiliência e otimismo. }\end{array}$ \\
\hline $\begin{array}{l}\text { Carolamgno et al. } \\
\text { (2014). }\end{array}$ & $\begin{array}{l}\text { Investigar a relação entre } \\
\text { desempenho profissional } \\
\text { e esperança, otimismo } \\
\text { e criatividade e testar } \\
\text { um modelo preditivo } \\
\text { do desempenho a partir } \\
\text { dessas variáveis. }\end{array}$ & $\begin{array}{l}\text { Estudo } \\
\text { quantitativo } \\
\text { transversal. }\end{array}$ & $\begin{array}{l}194 \\
\text { trabalhadores. }\end{array}$ & $\begin{array}{l}\text { Foram identificadas correlações } \\
\text { positivas entre desempenho, } \\
\text { esperança, otimismo e criatividade. } \\
\text { Esperaça e criatividade predizem o } \\
\text { desempenho no trabalho. }\end{array}$ \\
\hline
\end{tabular}


Tabela 1 (continuação)

Objetivo, delineamento, participantes e principais resultados dos estudos da revisão sistemática

\begin{tabular}{|c|c|c|c|c|}
\hline Autores (ano) & Objetivo & Delineamento & Participantes & Principais resultados \\
\hline $\begin{array}{l}\text { Davis et al. } \\
\text { (2014). }\end{array}$ & $\begin{array}{l}\text { Investigar a percepção } \\
\text { de estudantes sobre } \\
\text { esperança, escolhas de } \\
\text { carreira e obstáculos } \\
\text { relacionados a elas. }\end{array}$ & $\begin{array}{l}\text { Estudo } \\
\text { quantitativo } \\
\text { e qualitativo } \\
\text { transversal. }\end{array}$ & $\begin{array}{l}353 \\
\text { adolescentes. }\end{array}$ & $\begin{array}{l}\text { Meninos conversam mais com os } \\
\text { adultos sobre escolhas de carreira. } \\
\text { Metade dos participantes relatou que } \\
\text { existem barreiras para atingir suas } \\
\text { metas de carreira (poucos recursos, } \\
\text { fatores sociais e baixa qualidade } \\
\text { educacional). A maioria dos } \\
\text { participantes apresentou esperança } \\
\text { quanto ao futuro, sem diferenças } \\
\text { por sexo. }\end{array}$ \\
\hline Duffy et al. (2011). & $\begin{array}{l}\text { Examinar a relação entre } \\
\text { escolha vocacional e } \\
\text { satisfação acadêmica. }\end{array}$ & $\begin{array}{l}\text { Estudo } \\
\text { quantitativo } \\
\text { transversal. }\end{array}$ & $\begin{array}{l}312 \\
\text { universitários. }\end{array}$ & $\begin{array}{l}\text { Satisfação acadêmica foi predita por } \\
\text { calling, sendo a relação mediada } \\
\text { por esperança e percepção de } \\
\text { autoeficácia nas decisões de carreira. }\end{array}$ \\
\hline Hirschi (2014). & $\begin{array}{l}\text { Investigar a relação entre } \\
\text { esperança, planejamento } \\
\text { e decisão de carreira, } \\
\text { crenças de autoeficácia em } \\
\text { relação à carreira. Verificar } \\
\text { se essas variáveis mediam } \\
\text { a relação entre esperança e } \\
\text { comportamentos proativos } \\
\text { de carreira, satisfação com } \\
\text { a vida e com o trabalho. }\end{array}$ & $\begin{array}{l}\text { Estudo } \\
\text { quantitativo } \\
\text { transversal. }\end{array}$ & $\begin{array}{l}1334 \\
\text { universitários } \\
\text { e } 233 \\
\text { trabalhadores. }\end{array}$ & $\begin{array}{l}\text { Universitários: esperança foi } \\
\text { associada aos comportamentos } \\
\text { proativos e apresenta efeito direto e } \\
\text { indireto (mediada pelo planejamento, } \\
\text { decisão de carreira e autoeficácia) } \\
\text { com a satisfação com a vida. } \\
\text { Trabalhadores: esperança foi } \\
\text { associada indiretamente (mediada } \\
\text { pela clareza de autoconceito e dos } \\
\text { objetivos) com a satisfação com o } \\
\text { trabalho. }\end{array}$ \\
\hline $\begin{array}{l}\text { Hirschi et al. } \\
\text { (2015). }\end{array}$ & $\begin{array}{l}\text { Três estudos foram } \\
\text { realizados. } \\
\text { Estudos um e dois: } \\
\text { investigar a relação } \\
\text { entre comportamento } \\
\text { exploratório, esperança, } \\
\text { apoio social e crenças de } \\
\text { autoeficácia geral. } \\
\text { Estudo três: verificar a } \\
\text { relação entre esperança } \\
\text { e comportamento } \\
\text { exploratório ao longo de } \\
\text { um ano. }\end{array}$ & $\begin{array}{l}\text { Estudo } \\
\text { quantitativo } \\
\text { transversal } \\
\text { (estudos um } \\
\text { e dois) e } \\
\text { longitudinal } \\
\text { (estudo três). }\end{array}$ & $\begin{array}{l}\text { Estudo 1: } 228 \\
\text { adolescentes } \\
\text { em situação de } \\
\text { risco. } \\
\text { Estudo 2: } 223 \\
\text { universitários } \\
\text { do primeiro } \\
\text { ano. } \\
\text { Estudo 3: } 266 \\
\text { universitários. }\end{array}$ & $\begin{array}{l}\text { Estudos um e dois: o } \\
\text { comportamento exploratório pode } \\
\text { ser explicado pela esperança, pelo } \\
\text { apoio social e pelas crenças de } \\
\text { autoeficácia. } \\
\text { Estudo três: esperança e } \\
\text { comportamento exploratório } \\
\text { relacionam-se ao longo do tempo, } \\
\text { porém não foi possível demonstrar } \\
\text { a direção dos efeitos. }\end{array}$ \\
\hline $\begin{array}{l}\text { Kenny et al. } \\
(2010) \text {. }\end{array}$ & $\begin{array}{l}\text { Verificar a contribuição } \\
\text { da esperança, planos } \\
\text { de carreira e do apoio à } \\
\text { autonomia (professores } \\
\text { e supervisor) às crenças } \\
\text { relacionadas a conquistas } \\
\text { (ceticismo quanto à } \\
\text { contribuição da escola } \\
\text { para o sucesso futuro; } \\
\text { orientação para objetivos; } \\
\text { autoeficácia acadêmica). }\end{array}$ & $\begin{array}{l}\text { Estudo } \\
\text { quantitativo } \\
\text { transversal. }\end{array}$ & $\begin{array}{l}201 \\
\text { adolescentes. }\end{array}$ & $\begin{array}{l}\text { Esperança, planos de carreira e } \\
\text { apoio à autonomia explicaram } \\
37,5 \% \text { da variância das crenças } \\
\text { relacionadas a conquistas. } \\
\text { Esperança esteve relacionada } \\
\text { a todas as dimensões da } \\
\text { variável desfecho. }\end{array}$ \\
\hline
\end{tabular}


Tabela 1 (continuação)

Objetivo, delineamento, participantes e principais resultados dos estudos da revisão sistemática

\begin{tabular}{|c|c|c|c|c|}
\hline Autores (ano) & Objetivo & Delineamento & Participantes & Principais resultados \\
\hline $\begin{array}{l}\text { Malinowski \& } \\
\text { Lim (2015). }\end{array}$ & $\begin{array}{l}\text { Investigar a relação entre } \\
\text { mindfulness, engajamento } \\
\text { no trabalho e bem-estar. }\end{array}$ & $\begin{array}{l}\text { Estudo } \\
\text { quantitativo } \\
\text { transversal. }\end{array}$ & $\begin{array}{l}299 \\
\text { trabalhadores. }\end{array}$ & $\begin{array}{l}\text { Mindfulness prediz engajamento } \\
\text { no trabalho e bem estar geral. Essa } \\
\text { relação é mediada pelos afetos } \\
\text { positivos relacionados ao trabalho } \\
\text { e esperança, otimismo, resiliência e } \\
\text { autoeficácia. }\end{array}$ \\
\hline
\end{tabular}

\begin{tabular}{lll}
\hline Ouweneel et al. & Verificar relação entre & Estudo \\
(2012). & emoções positivas, & quantitativo \\
& esperança e engajamento & longitudinal. \\
& no trabalho.
\end{tabular}

$59 \quad$ Apresentar emoções positivas

Apresentar emoções positivas

rabalhadores. todos os dias teve efeito indireto

sobre o engajamento no trabalho, mediado pela esperança. Nível de esperança no início do dia foi predito pelas emoções positivas sentidas após o trabalho no dia anterior. Esperança esteve relacionada ao engajamento no trabalho.

\begin{tabular}{llll}
\hline Santilli et al. & Verificar a relação entre & Estudo & 120 \\
(2014). & esperança, adaptabilidade & quantitativo & trabalhadores \\
& e satisfação com a vida. & transversal. & $\begin{array}{l}\text { com deficiência } \\
\text { intelectual. }\end{array}$
\end{tabular}

Agência e rotas apresentaram correlação com as dimensões da intelectual carreira foi associada de maneira direta e indireta (através da esperança) à satisfação com a vida.

\begin{tabular}{ll}
\hline Schwartz et al. & Investigar o efeito da \\
(2007). & idade e do local de \\
& trabalho sobre o nível de \\
& esperança.
\end{tabular}

Estudo quantitativo transversal.

676 assistentes
676 assis
sociais.

Os trabalhadores do contexto público apresentaram mais esperança do que os do contexto privado. Para trabalhadores mais velhos, o nível de esperança foi o semelhante nos dois locais.

\begin{tabular}{|c|c|c|c|c|}
\hline $\begin{array}{l}\text { Sezgin \& Erdogan } \\
(2015) .\end{array}$ & $\begin{array}{l}\text { Explorar a influência } \\
\text { preditiva de otimismo } \\
\text { acadêmico, esperança } \\
\text { e zest (entusiasmo } \\
\text { para o trabalho) com a } \\
\text { percepção de autoeficácia } \\
\text { e de sucesso em } \\
\text { professores }\end{array}$ & $\begin{array}{l}\text { Estudo } \\
\text { quantitativo } \\
\text { transversal. }\end{array}$ & $\begin{array}{l}600 \\
\text { trabalhadores. }\end{array}$ & $\begin{array}{l}\text { Percepção de sucesso foi predito } \\
\text { por otimismo acadêmico, esperança } \\
\text { e zest (entusiasmo para o trabalho). } \\
\text { Autoeficácia foi predita por } \\
\text { percepção de sucesso, otimismo } \\
\text { acadêmico, esperança e zest } \\
\text { (entusiasmo para o trabalho). }\end{array}$ \\
\hline Sung et al. (2013). & $\begin{array}{l}\text { Verificar a validade do } \\
\text { Modelo integratitivo- } \\
\text {-contextual de desen- } \\
\text { volvimento de carreira; } \\
\text { explorar a relação entre } \\
\text { habilidades de carreira } \\
\text { (e.g. comportamento ex- } \\
\text { ploratório, autorregulação } \\
\text { da aprendizagem, uso do } \\
\text { apoio social), desfechos } \\
\text { educacionais e de car- } \\
\text { reira (e.g. autoeficácia, } \\
\text { identidade vocacional) e } \\
\text { esperança. }\end{array}$ & $\begin{array}{l}\text { Estudo } \\
\text { quantitativo } \\
\text { transversal. }\end{array}$ & $\begin{array}{l}132 \\
\text { universitários. }\end{array}$ & $\begin{array}{l}\text { O modelo integrativo-contextual } \\
\text { de desenvolvimento de carreira } \\
\text { apresentou validade na compreensão } \\
\text { de comportamentos de carreira } \\
\text { em universitários. Desfechos } \\
\text { educacionais e de carreira foram } \\
\text { preditos pelas habilidades de } \\
\text { carreira. Agência foi associada a } \\
\text { habilidades e desfechos de carreira. }\end{array}$ \\
\hline
\end{tabular}


Tabela 1 (continuação)

Objetivo, delineamento, participantes e principais resultados dos estudos da revisão sistemática

\begin{tabular}{|c|c|c|c|c|}
\hline Autores (ano) & Objetivo & Delineamento & Participantes & Principais resultados \\
\hline $\begin{array}{l}\text { Thompson et al. } \\
\text { (2014). }\end{array}$ & $\begin{array}{l}\text { Investigar a relação entre } \\
\text { variáveis pessoais e am- } \\
\text { bientais (experiência com } \\
\text { racismo, discriminação } \\
\text { por classe social, presença } \\
\text { de sintomas psicopatoló- } \\
\text { gicos, percepção de apoio } \\
\text { social, geração da família } \\
\text { que ingressa no ensino } \\
\text { superior) e esperança. }\end{array}$ & $\begin{array}{l}\text { Estudo } \\
\text { quantitativo } \\
\text { transversal. }\end{array}$ & $\begin{array}{l}176 \\
\text { universitários. }\end{array}$ & $\begin{array}{l}\text { Discriminação por racismo } \\
\text { e classe social, presença de } \\
\text { sintomas psicopatológicos foram } \\
\text { correlacionados negativamente com } \\
\text { esperança, enquanto que percepção } \\
\text { de apoio social foi correlacionada } \\
\text { positivamente. Esperança foi } \\
\text { predita por presença de sintomas } \\
\text { psicopatológicos e percepção de } \\
\text { apoio social. }\end{array}$ \\
\hline \multirow[t]{2}{*}{$\begin{array}{l}\text { Valero et al. } \\
(2015) \text {. }\end{array}$} & \multirow[t]{2}{*}{$\begin{array}{l}\text { Avaliar o papel da } \\
\text { esperança no desempenho } \\
\text { do trabalho e na intenção } \\
\text { de saída (turnover) da } \\
\text { formação profissional em } \\
\text { adolescentes. }\end{array}$} & \multirow[t]{2}{*}{$\begin{array}{l}\text { Estudo } \\
\text { quantitativo } \\
\text { transversal. }\end{array}$} & \multirow[t]{2}{*}{$\begin{array}{l}590 \\
\text { adolescentes } \\
\text { aprendizes. }\end{array}$} & $\begin{array}{l}\text { Esperança está relacionada de forma } \\
\text { positiva com objetivos autônomos, } \\
\text { afetos positivos no trabalho, } \\
\text { autoeficácia ocupacional, maior } \\
\text { desempenho no trabalho e menor } \\
\text { taxa de intenção de turnover. }\end{array}$ \\
\hline & & & & $\begin{array}{l}\text { Afetos positivos mediam efeito da } \\
\text { esperança sobre intenção de turnover } \\
\text { e desempenho no trabalho. Objetivos } \\
\text { autônomos mediam efeito da } \\
\text { esperança na intenção de turnover. }\end{array}$ \\
\hline $\begin{array}{l}\text { Yakushko \& } \\
\text { Sokolova (2010). }\end{array}$ & $\begin{array}{l}\text { Examinar a relação } \\
\text { entre esperança, } \\
\text { autoestima e influências } \\
\text { no desenvolvimento de } \\
\text { carreira de universitários. }\end{array}$ & $\begin{array}{l}\text { Estudo } \\
\text { quantitativo } \\
\text { transversal. }\end{array}$ & $\begin{array}{l}312 \\
\text { universitários. }\end{array}$ & $\begin{array}{l}\text { Autoestima foi correlacionada } \\
\text { positivamente com esperança e } \\
\text { com a percepção da importância da } \\
\text { educação para o desenvolvimento } \\
\text { de carreira. Pais foram as pessoas } \\
\text { que mais influenciaram nas decisões } \\
\text { de carreira. Participantes em que } \\
\text { suas escolhas de carreira foram } \\
\text { atribuídas a sua responsabilidade } \\
\text { em comparação a de seus pais, } \\
\text { apresentaram maior esperança. }\end{array}$ \\
\hline $\begin{array}{l}\text { Zhang et al. } \\
(2015) \text {. }\end{array}$ & $\begin{array}{l}\text { Investigar a relação entre } \\
\text { esperança, calling, sentido } \\
\text { de vida, satisfação com a } \\
\text { vida e decisão de carreira. }\end{array}$ & $\begin{array}{l}\text { Estudo } \\
\text { quantitativo } \\
\text { transversal. }\end{array}$ & $\begin{array}{l}518 \\
\text { universitários. }\end{array}$ & $\begin{array}{l}\text { Esperança media a relação entre } \\
\text { calling e sentido de vida, decisão de } \\
\text { carreira, e satisfação com a vida. }\end{array}$ \\
\hline $\begin{array}{l}\text { Wilkins et al. } \\
\text { (2014). }\end{array}$ & $\begin{array}{l}\text { Examinar a relação entre } \\
\text { esperança, otimismo e } \\
\text { adaptabilidade de carreira e } \\
\text { satisfação (domínios como } \\
\text { escola, relacionamentos, } \\
\text { tomadas de decisão, } \\
\text { percepção de apoio). }\end{array}$ & $\begin{array}{l}\text { Estudo } \\
\text { quantitativo } \\
\text { transversal. }\end{array}$ & $\begin{array}{l}242 \\
\text { adolescentes. }\end{array}$ & $\begin{array}{l}\text { Todas as dimensões da } \\
\text { adaptabilidade foram preditas } \\
\text { pela esperança. Apenas controle } \\
\text { e curiosidade foram preditas pelo } \\
\text { otimismo. Curiosidade e confiança } \\
\text { mediaram a relação entre esperança } \\
\text { e os componentes de satisfação. }\end{array}$ \\
\hline
\end{tabular}

\section{Definição teórica de esperança e instrumentos utilizados}

Foi possível identificar 16 artigos que utilizaram a definição teórica de esperança proposta por Snyder (2002). Dois artigos (Duffy et al., 2011; Yakushko \& Sokolova,
2010) utilizaram o construto "work hope" proposto por Juntunen e Wettersten (2006), compreendido como um estado emocional positivo direcionado ao trabalho e as metas relacionadas ao trabalho. O construto "work hope" é avaliado através da escala Work Hope Scale 
(Juntunen \& Wettersten, 2006), que busca relacionar a esperança proposta por Snyder (2002) com a carreira. Dessa forma, tem por objetivo avaliar a esperança para o trabalho, sendo que os itens propostos nesse instrumento foram construídos contemplando a definição de metas, rotas e agência de Snyder, porém foram redigidos para o contexto de trabalho (exemplo de item: Eu tenho um plano para conseguir ou manter um bom emprego ou carreira). Desse modo, é possível identificar o quanto a definição teórica proposta por Snyder (2002) tem sido utilizada para compreender esperança. Este aspecto está relacionado também ao maior número de estudos que utilizam as medidas construídas pelo autor e seus colaboradores.

Foi possível identificar que, para avaliar a esperança em adolescentes e adultos, as escalas utilizadas foram: a The Hope Scale construída por Snyder et al. (1991) utilizada em oito dos estudos (Buyukgoze-Kavas, 2016; Hirschi, 2014; Hirschi et al., 2015; Santilli et al., 2014; Sezgin \& Erdogan, 2015; Sung et al., 2014; Wilkins et al., 2014; Zhang et al., 2015); a State Hope Scale também proposta por Snyder et al. (1996) (Davis et al., 2014; Hirschi et al., 2015; Ouweneel et al., 2012; Schwartz et al., 2007) utilizado em 4 estudos; e a Work Hope Scale proposta por Juntunen e Wettersten (2006) (Duffy et al., 2011; Kenny et al., 2010; Thompson et al., 2014; Yakushko \& Sokolova, 2010) também utilizada em quatro trabalhos. A The Staats' Scale, construída por Staats e Stassen (1986) (Carlomagno, Natividade, Oliveira, \& Hutz, 2014), e o Psychological Capital Questionnaire, construída por Luthans, Avolio, Avey, e Norman (2007), foram utilizadas em um estudo cada uma (Malinowski \& Lim, 2015).

A The Hope Scale (Snyder et al., 1991) avalia a esperança disposicional (agência e rotas) em adultos, considerando a esperança como um traço do indivíduo, isto é, consistente no tempo (Snyder et al., 1991). Por outro lado, a State Hope Scale (Snyder et al., 1996) avalia a esperança (agência e rotas) como um estado apresentado pelo indivíduo em um dado momento no tempo. Tanto a esperança disposicional quanto a esperança como estado apresentam relação entre si, tendo em vista que um indivíduo com alta esperança disposicional tenderá a apresentar altos níveis de esperança de estado (Snyder et al., 1996). A Work Hope Scale (Juntunen \& Wettersten, 2006) avalia a esperança relacionada ao trabalho (metas, rotas e agência) e foi desenvolvida para adolescentes e adultos de grupos minoritários (por exemplo, dificuldades econômicas, baixo nível de escolaridade) (Juntunen \& Wettersten, 2006), baseada nos pressupostos de Snyder (2002). A The Staats 'Scale foi baseada nos postulados teóricos dos transtornos depressivos de Aaron Beck, sendo seu foco a dimensão cognitiva da esperança, compreendida a partir de dois fatores (hope-self e hope-other) que avaliam os desejos e expectativas em relação a si e aos outros (Staats, 1989). Por outro lado, a Psychological Capital Questionnaire (Luthans et al., 2007) avalia, além da esperança, construtos como autoeficácia, resiliência e otimismo em adultos. Os autores entendem que a esperança, bem como os demais constructos, compõem o capital psicológico do indivíduo, compreendido como motivações individuais desenvolvidas a partir dos construtos positivos acima descritos (Luthans et al., 2007).

Foram identificados poucos instrumentos que avaliavam a esperança em crianças e adolescentes nessa área de desenvolvimento de carreira. A Children's Hope Scale, desenvolvida por Snyder et al. (1997), foi utilizada em dois estudos (Hirschi et al., 2015; Valero et al., 2015). Embora esse instrumento possa ser utilizado para avaliar esperança disposicional (agência e rotas) em crianças e adolescentes, os artigos selecionados nesta análise que o utilizaram contaram com a participação somente de adolescentes.

Dentre essas medidas, a Work Hope Scale (WHS, Juntunen \& Wettersten, 2006) é o instrumento que têm maior proximidade com a carreira, tendo em vista que seus itens foram compostos associados a aspectos da vida profissional. As demais escalas avaliam o construto geral da esperança, associando-o a variáveis de carreira (como autoeficácia, comportamento exploratório, adaptabilidade de carreira, satisfação com o trabalho). No entanto, a WHS não foi tão utilizada quanto os instrumentos de avaliação de esperança geral desenvolvidos por Snyder et al. (1991, 1996). Possivelmente isso decorre do fato dessas medidas já terem sido validadas para outros países.

\section{Participantes e delineamentos dos estudos}

Nove estudos contaram com a participação de universitários (e.g. Thompson et al., 2014; Yakushko \& Sokolova, 2010), sete foram realizados com trabalhadores (e.g. Ouweneel et al., 2012; Santilli et al., 2014) e cinco com adolescentes (e.g. Hirschi et al., 2015; Kenny et al., 2010). É importante elucidar que o trabalho de Hirschi et al. (2015) foi composto por três estudos com diferentes delineamentos e amostras, sendo neste trabalho considerado como três estudos para fins de análise. Em especial, os resultados de Hirschi et al. (2015) denotam o quanto a esperança pode contribuir para os estudos relacionados à carreira em diferentes faixas etárias.

Ao investigar as contribuições da esperança em diferentes faixas etárias, foi possível identificar que, 
para os adolescentes, a mesma tem sido associada com o desenvolvimento de uma maior maturidade de carreira e de comportamentos exploratórios, além de promover confiança em relação às escolhas profissionais futuras (Hirschi et al., 2015; Kenny et al., 2010). No caso de estudantes universitários, a esperança pode auxiliar na satisfação acadêmica, visto que as metas estabelecidas por eles durante a graduação tendem a condizer com seus interesses profissionais (Buyukgoze-Kavas, 2016; Duffy et al., 2011). Para adultos trabalhadores, a esperança está relacionada ao desempenho no trabalho. Profissionais com escores mais altos de esperança parecem estar mais motivados para atingir as metas profissionais e, assim, criar diferentes rotas para atingir suas metas. Desta forma, parecem adaptar-se melhor diante de dificuldades, criando novas rotas, sem perder a motivação em busca da meta desejada (Carlomagno et al., 2014).

Dois estudos também investigaram grupos específicos da população tal como trabalhadores com deficiência intelectual (Santilli et al., 2014) e assistentes sociais (Schwartz et al., 2007). O estudo de Santilli et al. (2014) demonstrou que os componentes da adaptabilidade de carreira levam os indivíduos a pensar suas metas e rotas, além de contribuir na motivação para atingi-las. Esse resultado indica que, para trabalhadores com deficiência intelectual, torna-se relevante desenvolver intervenções com foco na adaptabilidade de carreira para, assim, estimular a reflexão sobre seus planos para o futuro, incentivando-os na sua capacidade de superação de obstáculos. De fato, indivíduos com mais esperança conseguem transpor com mais facilidade os obstáculos, mantendo a motivação; eles apresentam um maior número de metas em diferentes esferas ou papéis de vida e selecionam e buscam atingir metas mais difíceis (Snyder et al., 1991).

Quanto ao delineamento dos estudos analisados, 17 apresentaram um delineamento quantitativo e um apresentou delineamento misto. Dezesseis estudos tinham caráter transversal (e.g. Kenny et al., 2010) e dois longitudinais (Hirschi et al., 2015; Ouweneel et al., 2012). Destes, 17 foram estudos correlacionais (e.g. Buyukgoze-Kavas, 2016), um foi descritivo (Davis et al., 2014). A análise qualitativa do estudo de Davis et al. (2014) foi realizada a partir da leitura das respostas às questões abertas de um questionário, gerando temas que foram codificados e categorizados, a partir de análise temática. Através das questões abertas, os autores buscaram compreender as escolhas de carreira dos jovens bem como os obstáculos vivenciados por eles, contudo, o nível de esperança foi analisado somente através da escala State Hope Scale (Snyder et al., 1996). Diante da escassez de estudos longitudinais e qualitativos, a construção de pesquisas com tais delineamentos poderiam auxiliar na compreensão dos efeitos da esperança ao longo do desenvolvimento na carreira como, por exemplo, nas transições profissionais vividas pelo indivíduo, como transição universidade-trabalho, transições entre empregos e transição desemprego-emprego.

\section{Variáveis estudadas e principais resultados}

A partir dos estudos analisados, foi possível identificar que a esperança apresenta relação positiva com os construtos relacionados à carreira tais como desempenho no trabalho (Carlomagno et al., 2014; Ouweneel et al., 2012; Valero et al., 2015), adaptabilidade de carreira (Buyukgoze-Kavas, 2016; Santilli et al., 2014; Wilkins et al., 2014) e comportamento exploratório (Hirschi, 2014; Hirschi et al., 2015; Sung et al., 2013). A esperança também tem se mostrado associada a um planejamento de carreira mais definido e a uma melhor preparação para lidar com os desafios relacionados à carreira (Hirschi, 2014). Contudo, os construtos planejamento de carreira e esperança são similares, tendo em vista que um dos aspectos da esperança é o estabelecimento de metas. Desta forma, é esperado que sejam correlatos.

Além disso, a relação entre esperança e outros construtos tem sido investigada, tais como a satisfação com a vida, trabalho e satisfação acadêmica em universitários (Duffy et al., 2011; Hirschi, 2014; Santilli et al., 2014; Zhang et al., 2015). Estas variáveis podem estar associadas considerando que, a disposição para refletir sobre metas e construir planos profissionais pode estar associada à maior clareza do indivíduo sobre seus interesses e aspirações (Hirschi, 2014). Assim, o contexto de trabalho ou universitário pode ser um dos caminhos para atingir as metas estipuladas e a clareza do indivíduo sobre estes caminhos podem auxilia-lo a uma percepção positiva e, consequentemente, satisfação com a vida, trabalho ou contexto acadêmico. Enfrentar os problemas de maneira positiva também está associado à maior satisfação com a vida em universitários e a maiores níveis de esperança (agência e rotas) e crenças de autoeficácia (O’Sullivan, 2011).

Da mesma forma, elaborar planos de carreira parece motivar os indivíduos a desenvolver comportamento exploratório e atitudes proativas, uma vez que implica nas necessidades de conhecer a si, ao mercado de trabalho e as futuras opções profissionais, (Hirschi, 2014; Hirschi et al., 2015; Sung et al., 2013). De fato, a criação de planos está associada à definição de metas de carreira, sendo recomendado que sejam específicas e definidas ao longo do tempo - curto, médio e longo prazo (Sung et al., 2013). Ter clareza sobre o que se busca profissionalmente pode 
estar relacionado ao calling 2 , pois a identificação dos interesses possibilita o estabelecimento de metas e planos profissionais (Duffy et al., 2011; Zhang et al., 2015). No caso dos adolescentes, a presença da esperança os ajudou a compreender a relevância da escola para o futuro, sendo esta instituição percebida como uma das rotas para atingir as metas elencadas (Kenny et al., 2010).

Embora a maior parte dos estudos selecionados nesta revisão evidenciaram a importância das metas e rotas, o estudo de Sung et al. (2013) encontrou que a agência foi o único componente da esperança que apresentou associação com as variáveis relacionadas à carreira (tais como comportamento exploratório, identidade vocacional e autoeficácia). Os achados evidenciaram que a agência deve ser foco de intervenções com estudantes universitários, pois pode auxiliar no desenvolvimento de carreira dos mesmos no contexto acadêmico e nas mudanças e transições de carreira (Sung et al., 2013). Desenvolver aspectos relacionados à agência pode auxiliar a diminuir a insegurança nas tomadas de decisões (Mc Dermott et al., 2015).

Outras variáveis abordadas nos artigos revisados foram o apoio social e as influências, diretas ou indiretas, de professores e de familiares nas questões profissionais (Hirschi et al., 2015; Sung et al., 2013; Thompson et al., 2014, Vela et al., 2014; Yakushko \& Sokolova, 2010). No entanto, a percepção de que o indivíduo é o principal responsável por suas escolhas está relacionado com a motivação para atingir as metas, pois jovens que descreveram seus objetivos de carreira mais associados aos desejos de suas famílias apresentaram menores níveis de esperança (Yakushko \& Sokolova, 2010).

Por outro lado, mesmo que um indivíduo seja responsável por suas próprias escolhas, a presença de variáveis contextuais como discriminação por racismo e classe social foram associadas de forma negativa à esperança, enquanto o apoio social foi associado de forma positiva (Thompson et al., 2014). Assim, ressalta-se a necessidade de intervenções que procurem desenvolver a esperança, principalmente em grupos que se encontram em situações de vulnerabilidade. As intervenções devem focar na percepção do apoio social, uma vez que alguns dos obstáculos podem estar relacionados ao baixo apoio familiar às escolhas profissionais (Davis et al., 2014; Thompson et al., 2014). A presença de redes de apoio auxilia aos jovens no estabelecimento de rotas e na motivação para atingir suas metas (Yakushko \& Sokolova, 2010). Criar espaços que estimulem a reflexão sobre os interesses relacionados ao futuro e clarificar metas pode trazer impacto para a retenção dos estudantes nas escolas, principalmente em grupos minoritários, pois lhes ajuda a perceber um sentido no processo de escolarização (Park-Taylor \& Vargas, 2012). Desta forma, desenvolver programas que busquem estimular a resolução de problemas, associados aos componentes da esperança, pode auxiliar aos indivíduos no desenvolvimento de um maior engajamento acadêmico (Davidson et al., 2012; O'Sullivan, 2011) e em uma maior percepção de eficácia para antecipar e enfrentar obstáculos em adolescentes (Wilkins et al., 2014).

\section{Considerações finais}

Este estudo buscou caracterizar estudos empíricos que relacionaram esperança e carreira a partir de uma revisão sistemática da literatura. Embora seja um aspecto estudado há, pelo menos, duas décadas (Snyder et al., 1991), recentemente tem sido associado ao desenvolvimento de carreira. Os resultados encontrados nesta revisão demonstram que a esperança é uma variável que influencia no desenvolvimento de carreira em diferentes etapas do ciclo vital. Através dela, é possível estabelecer metas e rotas e ter motivação para empregar comportamentos na obtenção das metas. A clareza das metas está relacionada ao melhor desempenho acadêmico e profissional, além de ser encontrada em indivíduos que demonstram estar mais satisfeitos com sua vida e com seu trabalho.

Os resultados associados à carreira parecem apontar para achados mais genéricos, talvez pelo fato de utilizar instrumentos, também genéricos, para avaliar esperança. Desta forma, torna-se relevante investigar de forma mais específica a relação entre esperança e carreira. Para tanto, sugere-se a realização de estudos longitudinais e qualitativos, principalmente com indivíduos em transição de carreira, a fim de conhecer o papel da esperança em situações críticas experimentadas ao longo da carreira. Estudos qualitativos poderão auxiliar na investigação da dinâmica da esperança, buscando conhecer como indivíduos, com diferentes níveis de esperança, vivenciam a superação de obstáculos e a construção de novos planos de carreira. Além disso, é preciso verificar a associação de esperança com clareza de autoconhecimento, visto que é importante que as metas façam sentido para os indivíduos. Também, sugere-se a construção de estudos voltados para crianças

\footnotetext{
${ }^{2}$ Define-se a partir de três aspectos: (a) motivação para o trabalho advém a partir de uma fonte externa - por exemplo, necessidade social, (b) consonância entre o propósito de vida e o propósito de trabalho e (c) percepção do indivíduo de que seu trabalho é importante para a sociedade (Dik \& Duffy, 2009; Duffy et al., 2011).
} 
e adolescentes, buscando identificar a contribuição da esperança para o envolvimento na escola e em atividades extracurriculares, por exemplo, no caso das crianças e compreender como a esperança influencia nas escolhas de carreira dos adolescentes.

Além disso, torna-se importante a construção de intervenções com foco na esperança. Para além do uso de técnicas que desenvolvam somente metas e planos de carreira, torna-se relevante utilizar técnicas que visem a promoção do autoconhecimento, clarificando as habilidades e competências relacionadas à carreira. Tais programas poderiam beneficiar indivíduos em diferentes etapas de sua carreira tais como adolescentes, pessoas desempregadas, estudantes universitários, trabalhadores, indivíduos em vulnerabilidade social e aqueles que estão vivenciando transições de carreira.

Para além destes aspectos, esta revisão também apresenta algumas limitações. A primeira delas é o mapeamento de publicações de somente cinco bases de dados, duas nacionais e três internacionais. Logo, é possível que outros artigos sobre a temática tenham ficado de fora desta revisão. A partir disso, recomenda-se a realização de revisões em bases de dados diferentes, de preferência em outros idiomas além do Português e Inglês, como o Espanhol. Outra limitação refere-se à ausência de seis artigos selecionados pelo resumo, cujos textos completos estavam indisponíveis. A não inclusão de artigos de revisão de literatura e de tradução, adaptação e validação de instrumentos também constitui uma limitação na medida em que poderiam contribuir para a compreensão teórica da esperança e sua relação com a carreira. Além disso, é possível que outras pesquisas sobre a temática da esperança não tenham sido localizadas com as palavras-chave "esperança" ou "hope", visto que os componentes da esperança, como metas (ou objetivos), rotas e planos, podem ter sido abordados sem a denominação de esperança.

\section{Referências}

Amundson, N., Niles, S., Yoon, H., Smith, B., I, H., \& Mills, L. (2013). Hope-Centered Career development for university/ college students. Final report project.

Bandura, A. (1977). Self-efficacy: Toward a unifying theory of behavior change. Psychological Review, 84, 191-215. DOI: http://dx.doi.org/10.1037/0033-295X.84.2.191

Bandura, A. (2001). Social cognitive theory: An agentic perspective. Annual Reviews Psychology, 52, 1-26. DOI: 10.1146/ annurev.psych.52.1.1

*Buyukgoze-Kavas, A. (2016). Predicting career adaptability from positive psychological traits. The Career Development Quarterly, 64, 114-125. DOI: 10.1002/cdq.12045

Bernes, K., Bardick, A., \& Orr, D. (2007). Career guidance and counselling efficacy studies: An international research agenda. International Journal for Educational and Vocational Guidance, 7, 81-96. DOI: 10.1007/s10775-007-9114-8

*Carlomagno, L., Natividade, J., Oliveira, M., \& Hutz, C. (2014). Relações entre Criatividade, Esperança, Otimismo e Desempenho Profissional. Temas em Psicologia, 22(2), 497-508. DOI: 10.9788/TP2014.2-18

Costa, A. B., \& Zoltowski, A. P. C. (2014). Como escrever um artigo de revisão sistemática. In: S H. Koller, M. C. Couto, \& J. Hohendorff. (Eds.). Manual de Produção Científica (p. 55-70). Porto Alegre: Penso.

Davidson, O., Feldman,D., \& Margalit, M.(2012). Afocused intervention for 1st-year college studentes: Promotinhope, sense of coherence, and self-efficacy. The Journal of Psychology, 146(3), 333-352. DOI: 10.1080/00223980.2011.634862

*Davis, J., Dodge, E., \& Welderufael, M. (2014). Intercultural partnering for the benefit of South Africa town ship high school students. Occupational Therapy International, 21, 186-195. DOI: 10.1002/oti.1379

Dik, B. J., \& Duffy, R. D. (2009). Calling and vocation at work: Definitions and prospects for research and practice. The Counseling Psychologist, 37, 424-250. DOI:10.1177/0011000008316430.

*Duffy, R., Allan, B., \& Dik, B. (2011). The presence of a calling and academic satisfaction: Examining potential mediators. Journal of Vocational Behavior, 79, 74-80. DOI:10.1016/j.jvb.2010.11.001

Feldman, D., \& Kubota, M. (2015). Hope, self-efficacy, optimism, and academic achievement: Distinguishing constructs and levels of specificity in predicting college grade-point average. Learning and Individual Differences, 37, 210-216. DOI: 10.1016/j.lindif.2014.11.022

*Hirschi, A. (2014). Hope as a resource for self-directed career management: Investigating mediating effects on proactive career behaviors and life and job satisfaction. Journal of Happiness Study, 15, 1495-1512. DOI: 10.1007/ s10902-013-9488-x

*Hirschi, A., Abessolo, M., \& Froidevaux, A. (2015). Hope as a resource for career exploration: Examining incremental and cross-lagged effects. Journal of Vocational Behavior, 86, 38-47. DOI: 10.1016/j.jvb.2014.10.006 
Jordaan, J. P. (1963). Exploratory behaviour: The formation of self and occupational concepts. In D. E. Super, R. Starshevsky, R. Matlin, \& J. P. Jordan (Eds.). Career development: Self-concept theory. New York: College Entrance Board.

Juntunen, C., \& Wettersten, K. (2006). Work hope: Development and initial validation of a measure. Journal of Couseling Psychology, 53(1), 94-106. DOI: 10.1037/0022-0167.53.1.94

*Kenny, M., Walsh-Blair, L., Blustein, D., Bempechat, J., \& Seltzer, J. (2010) Achievement motivation among urban adolescents: Work hope, autonomy support, and achievement-related beliefs. Journal of Vocational Behavior, 77, 205-212. DOI: $10.1016 /$ j.jvb.2010.02.005

Lima-Dias, M., \& Soares, D. H. (2012). Planejamento de carreira: Uma orientação para universitários. Psicologia e Argumento, 30(68), 53-61.

Luthans, F., Avolio, B. J., Avey, J. B., \& Norman, S.M. (2007). Positive psychological capital: measurement and relationship with performance and satisfaction. Personnel Psychology, 60(3), 541-572. DOI:10.1111/j.1744-6570.2007.00083.x.

*Malinowski, P., \& Lim, H. (2015). Mindfulness at work: positive affect, hope, and optimism mediate the relationship between dispositional mindfulness, work engagement, and well-being. Mindfulness, 6(6), 1250-1262. DOI: 10.1007/ s12671-015-0388-5

Mc Dermott, R., Cheng, H., Wright, C., Browning, B., Upton, A., \& Sevig, T. (2015). Adult attachment dimensions and college student distress: The mediating role of hope. The Counseling Psychologist, 43(6) 822-852. DOI: $10.1177 / 0011000015575394$

Niles, S. (2011). Career flow: A hope-centered model of career development. Journal of Employment Counseling, 48, 173-175. DOI: $10.1002 / j .2161-1920.2011 . t b 01107 . x$

Onwuegbuzie, A. J., \& Snyder, C. R. (2000). Relations between hope and graduate students' studying and test-taking strategies. Psychological Reports, 86, 803-806. DOI: 10.2466/pr0.2000.86.3.803

O'Sullivan, G. (2011). The relationship between hope, eustress, self-efficacy, and life satisfaction among undergraduates. Social Indicators Research, 101, 155-172. DOI: 10.1007/s11205-010-9662-z

*Ouweneel, E., Blanc, O. M., Schaufeli, W., Wijhe, C. (2012). Good morning, good day: A diary study on positive emotions, hope, and work engagement. Human Relations, 65(9), 1129-1154. DOI:10.1177/0018726711429382

Park-Taylor, J., \& Vargas, A. (2012). Using the constructs multifinality, work hope, and possible selves with urban minority youth. The Career Development Quarterly, 60, 243-253. DOI: 10.1002/j.2161-0045.2012.00020.x

*Santilli, S., Nota, L., Ginevra, M., \& Soresi, S. (2014). Career adaptability, hope and life satisfation in workers with intelectual disability. Journal of Vocational Behavior, 85, 67-74. DOI: 10.1016/j.jvb.2014.02.01

Savickas, M. (2013). Career construction theory and practice. In R. W. Lent \& S. D. Brown (Eds.), Career development and counseling: Putting theory and research to work (pp 147-183). Hoboken, New Jersey: John Wiley and sons.

Savickas, M. L. (1997). Career adaptability: An integrative construct for life-span, life-space theory. Career Development Quarterly, 45(3), 247-259. DOI: 10.1002/j.2161-0045.1997.tb00469.x

Savickas, M., Nota, L., Rossier, J., Dauwalder, J., Duarte, M., Guichard, J., Soresi, S., ... \& Vianen, A. (2009) Life designing: A paradigm for career construction in the 21st century. Journal of Vocational Behavior 75, 239-250. DOI:10.1016/j.jvb.2009.04.004

*Schwartz, R., Tiamiyu, M., \& Dwyer, D. (2007). Social worker hope and perceived burnout: the effects of age, years in practice, and setting. Administration in Social Work, 31(4), 103-119. DOI: 10.1300/J147v31n04_08

Seirup, H., \& Rose, S. (2011). Exploring the effects of hope on GPA and retention among college undergraduate students on academic probation. Education Research International, 2011, 1-7. DOI: 10.1155/2011/381429

*Sezgin, F., \& Erdogan, O. (2015).Academic optimism, hope and zest for work as predictors of teacher self-efficacy and perceived success. Educational Sciences: Theory \& Practice, 15(1), 7-19. DOI: 10.12738/estp.2015.1.2338

Snyder, C., Harris, C., Anderson, J. R., Holleran, S. A., Irving, L. M., Sigmon, S. T., \& Harney, P. (1991). The will and the ways: Development and validation of an individual differences measure of hope. Journal of Personality and Social Psychology, 60(4), 570-585.

Snyder, C., Sympson, S. C., Ybasco, F. C., Borders, T. F., Babyak, M. A., \& Higgins, R. L. (1996). Development and validation of the State Hope Scale. Journal of Personality and Social Psychology, 70(2), 321-335.

Snyder, C. R., Hoza, B., Pelham, W. E., Rapoff, M., Ware, L., Danovsky, M., \& Stahl, K. J. (1997). The development and validation of the Children's Hope Scale. Journal of Pediatric Psychology, 22(3), 399-421. DOI: https://doi. org/10.1093/jpepsy/22.3.399 
Snyder, C. (2002). Hope theory: Rainbows in the mind. Psychological Inquiry, 13(4) 249-275. DOI: http://dx.doi. org/10.1207/S15327965PLI1304_01

Snyder, C., Shorey, H., Cheavens, J., Pulvers, K., Adams III, V., \& Wiklund, C. (2002). Hope and Academic Success in College. Journal of Educational Psychology, 94(4), 820-826. DOI: 10.1037//0022-0663.94.4.820

Staats, S., \& Stassen, M. A. (1986). The Hope Index: A measure of self-other-world expectations for adults. Oral communication conducted at the 94th Annual Convention of the American Psychological Association at the Ohio State University: Newark campus, Columbus, Ohio.

*Sung, Y., Turner, S., \& Kaewchinda, M. (2013). Career development skills, outcomes, and hope among college students. Journal of Career Development, 40(2), 127-145. DOI: 10.1177/0894845311431939

*Thompson, M., Her, P., \& Nitzarim, R. (2014). Personal and contextual variables related to work hope among undergraduate students from underrepresented backgrounds. Journal of Career Assessment, 22(4), 595-609. DOI: $10.1177 / 1069072713514814$

*Valero, D., Hirschi, A., \& Strauss, K. (2015). Hope in adolescent careers: Mediating effects of work motivation on career outcomes in swiss apprentices. Journal of Career Development, 42(5) 381-395. DOI: 10.1177/0894845314566866

Vela, J., Lerma, E., Lenz, A., Hinojosa, K., Hernanzez-Duque, O., \& Gonzalez, S. (2014). Positive psychology and familial factors as predictors of latina/o students' hope and college performance. Hispanic Journal of Behavioral Sciences, 36(4), 452-469.

*Yakushko, O., \& Sokolova, O. (2010). Work hope and influences of the career development among Ukrainian college students. Journal of Career Development, 36(4), 310-323. DOI: 10.1177/0894845309345670

*Wilkins, K., Santilli, S., Ferrari, L., Nota, L., Tracey, T., \&Soresi, S. (2014). The relationship among positive emotional dispositions, career adaptability, and satisfaction in Italian high school students. Journal of Vocational Behavior, 85, 329-338. DOI: $10.1016 /$ j.jvb.2014.08.004

Whiston, S, (2011). Vocational counseling and interventions: An exploration of future "big" questions. Journal of Career Assessment, 19(3), 287-295. DOI: 0.1177/1069072710395535

*Zhang, C., Herrmann, A., Hirschi, A., Wei, J., \& Zhang, J. (2015). Assessing calling in chinese college students: Development of a measure and its relation to hope. Journal of Career Assessment, 23(4) 582-596. DOI: $10.1177 / 1069072715595804$

Recebido: $10 / 05 / 2017$

$1^{a}$ reformulação: $31 / 01 / 2018$

Aceite final: 07/02/2018

Sobre os autores

Cássia Ferraza Alves é psicóloga pelo Centro Universitário Franciscano (UNIFRA) e mestre em Psicologia pela Universidade Federal do Rio Grande do Sul (UFRGS). Atualmente, é doutoranda no Programa de Pós-graduação em Psicologia da UFRGS e docente do Centro Universitário da Serra Gaúcha - FSG.

Clarissa Tochetto de Oliveira é psicóloga e mestre em Psicologia pela Universidade Federal de Santa Maria (UFSM) e doutora em Psicologia pela Universidade Federal do Rio Grande do Sul (UFRGS).

Ana Cristina Garcia Dias é psicóloga, mestre em Psicologia pela UFRGS, doutora em Psicologia pela Universidade de São Paulo (USP) e professora do Programa de Pós-graduação em Psicologia da UFRGS.

Marco Antônio Pereira Teixeira é psicólogo, mestre e doutor em Psicologia pela UFRGS e professor do Programa de Pós-graduação em Psicologia da UFRGS. 